\title{
Design A Distributed Amplifier System Using I-Filtering Structure
}

\author{
Azad Raheem Kareem \\ University of Technology, Control and Systems Eng. Dept. Baghdad, Iraq \\ E-Mail: drazadnarj@ hotmail.com
}

\begin{abstract}
This paper reviews in brief the principle of distributed amplifier with its classical structure. Alternation to the basic design including the use of $\pi$-filtering sections instead of Tsections in gate and drain lines is proposed. The new design method has the advantages of much gain flatness near the cutoff frequency and less inductances at the corners of the amplifier. These are great benefits make the new design to be better than the previous ones especially in modeling the amplifier in integrated circuit technology. The distributed amplifier is used in microwave applications.
\end{abstract}

Keywords - Microwave circuits, microwave filters, distributed amplifiers.

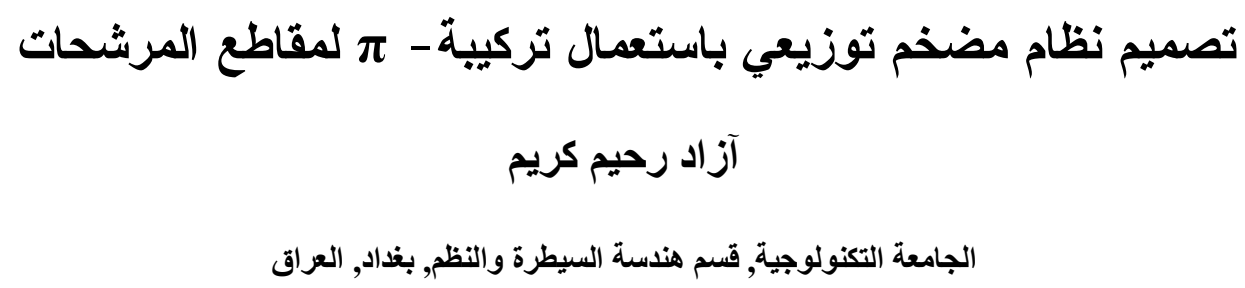

الخلاصة

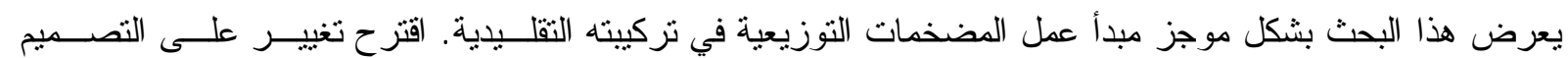

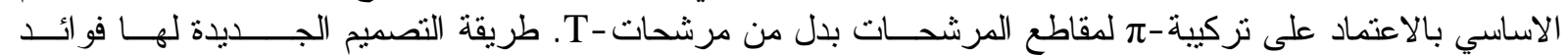

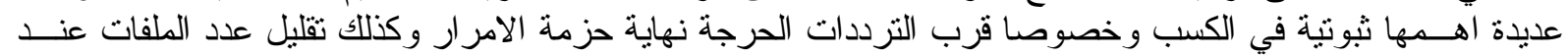

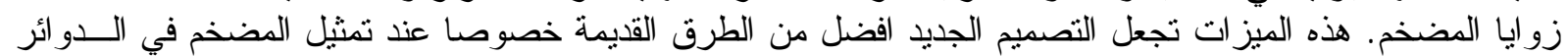
المتكاملة. ان المضخم التوزيعي يستخدم في التطبيقات المايكروية. 


\section{Introduction}

The rapid progress in microwave communication, such as wireless networks and satellite systems, drives the demands for wideband amplifiers with multi-gigahertz bandwidth. Distributed amplifiers (DAs) have always been the best choices for this purpose [1]-[3]. Its concept is based on connecting several transistors in a manner between two artificial transmission lines whereby the input and output parasitic capacitances of the transistors $\left(\mathrm{C}_{\mathrm{gs}}\right.$ and $\mathrm{C}_{\mathrm{ds}}$ ) are separated and absorbed into LPF segments of the transmission lines. As a result, the band of operation of the transistors will be infinite and the overall bandwidth of the circuit be the cutoff frequency of the filters. In this way, it is possible to achieve an amplifier with bandwidth greater than that achieved by a simple cascaded amplifier.

Distributed amplifiers nowadays are implemented using monolithic microwave integrated circuit (MMIC) technology [4]-[7]. One of the main challenges in realizing a fully integrated distributed amplifier is creating the high-quality inductors necessary. Also, the value of the integrated circuit inductor depends on its layout [8], therefore it is a big gain if the number and the values of the inductors in the circuits are reduced.

This paper will demonstrate a new design method for distributed amplifiers. The classical structure of the artificial transmission lines which is based on cascaded T-section LPFs and terminated by bisected- $\pi$ m-derived matching network will be replaced by cascading $\pi$-section LPFs and using bisected-T m-derived network for source and load matching. The goal is to reduce the inductances in the circuit and then to improve the amplifier performance.

\section{Basic Da Structure}

Fig. 1 shows the schematic diagram of a four-stage distributed amplifier. On the input side, inductors $\mathrm{L}_{\mathrm{g}}$ are placed between the gate-to-source capacitances $\mathrm{C}_{\mathrm{gs}}$ of the adjacent transistors. On the other side, inductors $\mathrm{L}_{\mathrm{d}}$ are placed between drain-to-source capacitances. Thus, the familiar lumped-element artificial transmission lines are formed. They can be named as gate and drain lines. The two lines are coupled by the transconductances $\left(g_{m}\right)$ of the active devices.

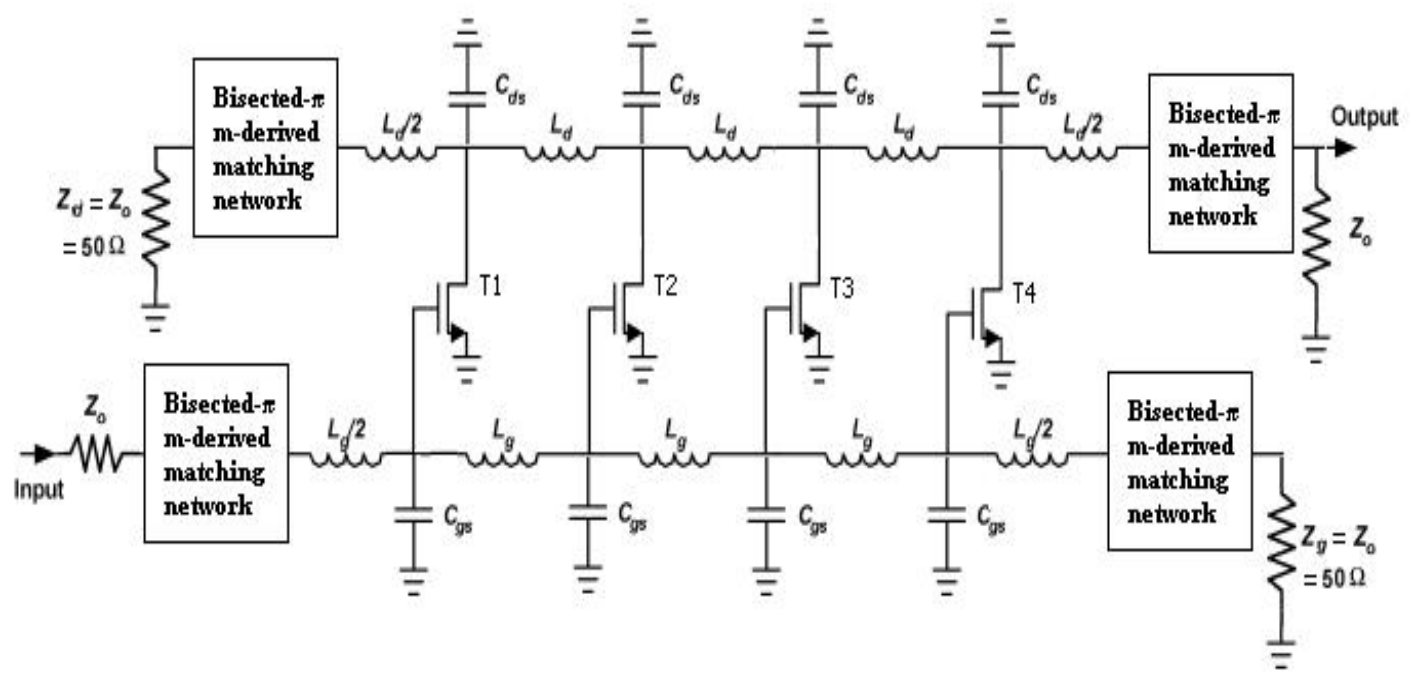

Fig.1 Basic structure of a 4-stage DA

The input signal applied to the input port of the gate line travels down the line to the terminated end $\left(Z_{g}\right)$ where it is absorbed. The traveling signal is picked up by the gates of the individual transistor and transferred to the drain line via their transconductances. The signals 
on the drain line add in the forward direction as they arrive at the output. The available power gain of the amplifier is given by [2]

$$
G=\left(g_{m}\right)^{2} Z_{d} Z_{g} n^{2} / 4
$$

where $n$ is the number of stages.

The basic topology of the amplifier is based on cascading constant-k, T-section filters on the drain and gate lines with identical characteristic impedances $\left(Z_{o}\right)$. The bandwidth of the circuit is determined by the cutoff frequency of the filters. If $\mathrm{L}_{\mathrm{g}}=\mathrm{L}_{\mathrm{d}}=L$, and $\mathrm{C}_{\mathrm{gs}}=\mathrm{C}_{\mathrm{ds}}=C$ (note: since $C_{d s}$ is much smaller than $C_{g s}$, it is necessary to add a parallel capacitance to the drain circuit of each stage), then the bandwidth and the characteristic impedance of the lines are given by

$$
\begin{aligned}
& \text { B.W. }=2 / \sqrt{L C} \\
& Z_{o}=\sqrt{L / C}
\end{aligned}
$$

To achieve an impedance match over a broad range, the bisected- $\pi$, m-derived section shown in Fig. 2 must be used on the four corners of the amplifier circuit. The feature of the mderived network is to transfer the line impedance into the source and load impedance $\left(Z_{o}\right)$. The value of $m=0.6$ provides near optimal results for this purpose [9].

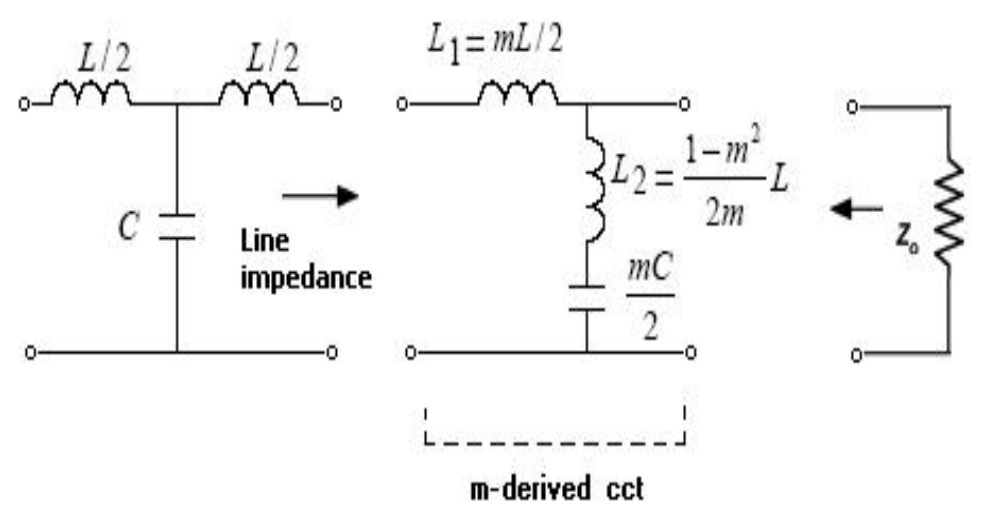

Fig. 2 Bisected- $\pi$ m-derived matching network

Such matching network increases the die area and the complexity of overall circuit design and optimization.

\section{New Structure For Das}

The image parameter method of the filter design may be applied to the distributed amplifier since it consists of a cascade of identical two-port networks constructing its artificial transmission lines. Conventional distributed amplifiers usually use $\mathrm{T}$-sections as the basic iterative two-port networks in the gate and drain circuits. The reducing of the total number of the inductors or their values is one of the necessary conditions of amplifier implementation and tuning in ICs. Therefore, $\pi$-network may also be used for transmission lines in the distributed amplifier design. The new structure of the amplifier is shown in Fig.3. in this circuit, the inductors $\left(\mathrm{L}_{\mathrm{g}} / 2\right)$ and $\left(\mathrm{L}_{\mathrm{d}} / 2\right)$ at the four terminals of the circuit are omitted. This is the first advantage of the new method. 


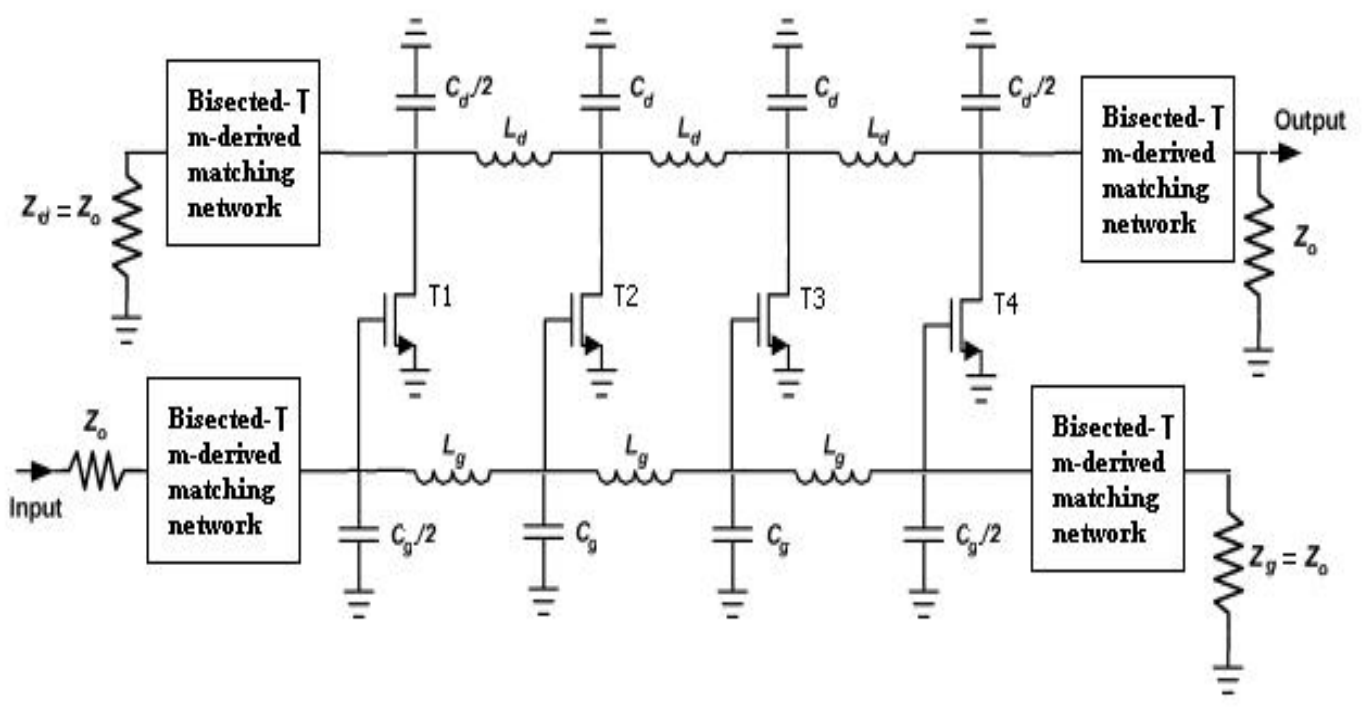

Fig.3 Proposed structure of a 4-stage DA

\section{A. Circuit Analysis}

The only change in the circuit topology is occurred on the passive elements ( $L$ and $C$ ). These elements have no effect on the gain computation, thus equation (1) can be used to calculate the power gain of the proposed amplifier. On the plus side, equations (2) and (3) can also be used here for $B . W$. and $Z_{o}$ calculations since the low-pass filter sections in $\mathrm{T}$ and $\pi$ forms have the same cutoff frequency and characteristic impedance [10].

\section{B. Image Impedance Matching}

The image impedance $\left(Z_{i}\right)$ of a symmetric two-port network is defined as the impedance looking into port 1 when port 2 is terminated in $Z_{i}$ or vice versa [10]. For a lossless $\pi$ section LPF as shown in Fig.4, the image impedance is

$$
Z_{i \pi}=\sqrt{\frac{L}{C}} / \sqrt{1-\left(\frac{\omega}{\omega_{c}}\right)^{2}}
$$

Thus, for $\omega \ll \omega_{c}$ the filter structure has a real image impedance, $Z_{i \pi} \rightarrow \sqrt{L / C}$, which is equal to its characteristic impedance and to the $50 \Omega \mathrm{load} /$ source impedance. But for frequencies near the cutoff, the image impedance is not constant and frequency dependent. Consequently, the lines will not match the load and source impedances which cause a deviation in the ideal behavior of the amplifier.

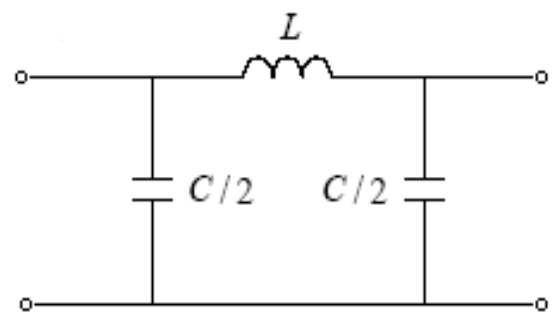

Fig.4 $\pi$-section filter

The bisected-T m-derived filter section of Fig.5 is a modification of the constant-k section proposed to overcome this problem. 


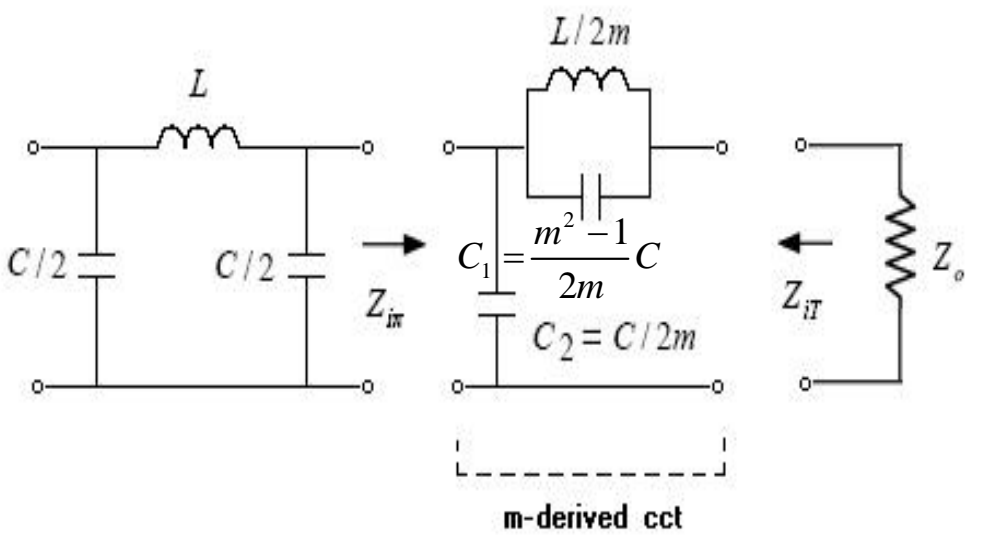

fig.5 Proposed matching network

The image impedance at the input port of this circuit is equal to the image impedance of the line sections $Z_{i \pi}$, but at the output port the image impedance is equal to

$$
Z_{i T}=Z_{o} \frac{\sqrt{1-\left(\frac{\omega}{\omega_{c}}\right)^{2}}}{\left[1-\left(1-\frac{1}{m^{2}}\right)\left(\frac{\omega}{\omega_{c}}\right)^{2}\right]}
$$

where $m$ is a factor can be used to minimize the deviation of $Z_{i T}$ over the passband of the filter and to match the load impedance $\left(Z_{o}\right)$. Fig. 6 shows the variation of $Z_{i T}$ with frequency for several values of $m$. As evident from the figures, the value of $m=1.7$ gives the best results.

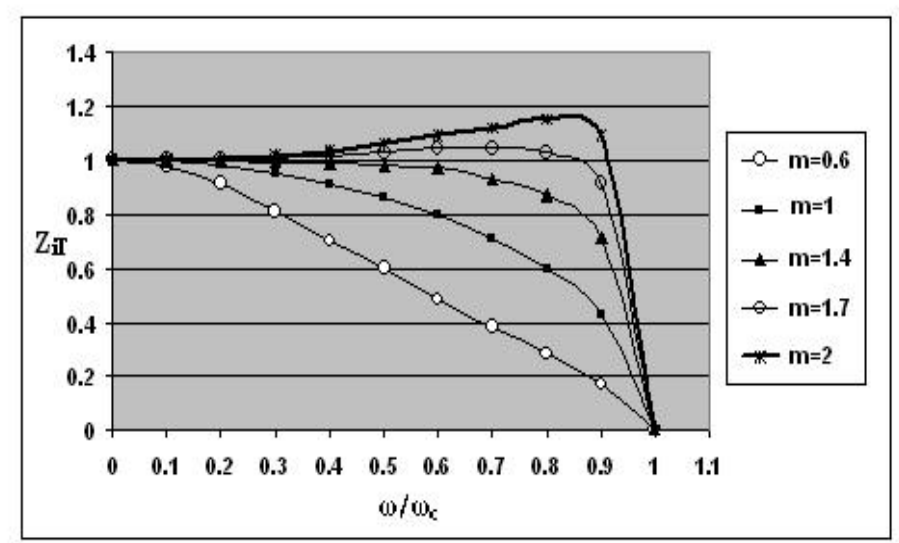

Fig.6 Variation of $\mathrm{Z}_{\mathrm{iT}}$ for different values of $m$

The bisected-T m-derived filter section is better than bisected- $\pi \mathrm{m}$-derived filter since it takes up less layout area due to the use of just one inductor. This is another advantage for the new amplifier. The performance of the design method is validated through the following example. 


\section{Al-Rafidain Engineering Vol.17 No.3 \\ June 2009 \\ Design Example}

Using topologies based on $\mathrm{T}$ and $\pi$-filtering, two four-stage DAs have been designed for $14 \mathrm{~dB}$ gain and $10 \mathrm{GHz}$ bandwidth. The inductance and capacitance values of the constant-k filter section in both circuits are $L=1.6 \mathrm{nH}$ and $C=0.636 \mathrm{pF}$. The elements of the bisected- $\pi$ matching network in the first circuit are $\left(L_{1}=0.48 \mathrm{nH}, L_{2}=0.53 \mathrm{nH}\right.$, and $\left.C=0.2 \mathrm{pF}\right)$, while the elements of the bisected-T network in the second circuit are $\left(L=0.47 \mathrm{nH}, C_{l}=0.35 \mathrm{pF}\right.$, and $\left.C_{2}=0.18 \mathrm{pF}\right)$. The transconductance value of the transistors in both circuits is $\left(g_{m}=0.05 \Omega^{-1}\right)$. The characteristic impedance $\left(Z_{o}\right)$ for the circuits is $50 \Omega$. The schematic DAs were simulated using Microwave Office software and the S-parameter results of the two circuits are given in Fig.7 and Fig.8. The simulations are down without optimization process. It is clear that the gain response $\left(S_{21}\right)$ is more flatted and the input/output return loss $\left(S_{11}\right.$ and $\left.S_{22}\right)$ are reduced and improved by using the new $\pi$-filtering method especially near the cutoff frequency.

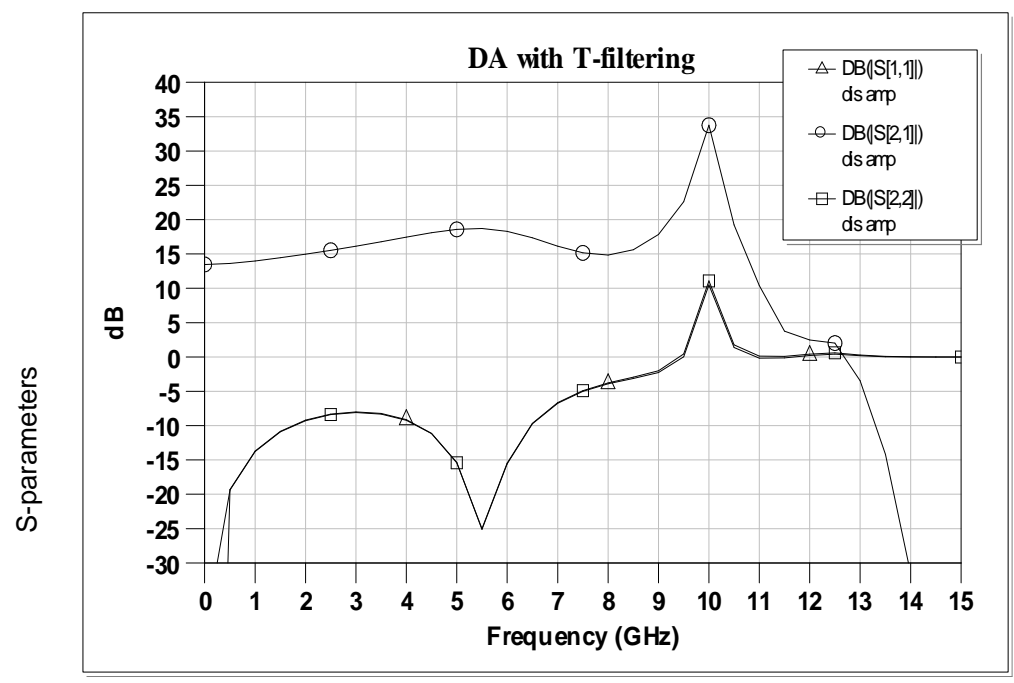

Fig.7 S-parameters of the DA based T-filtering

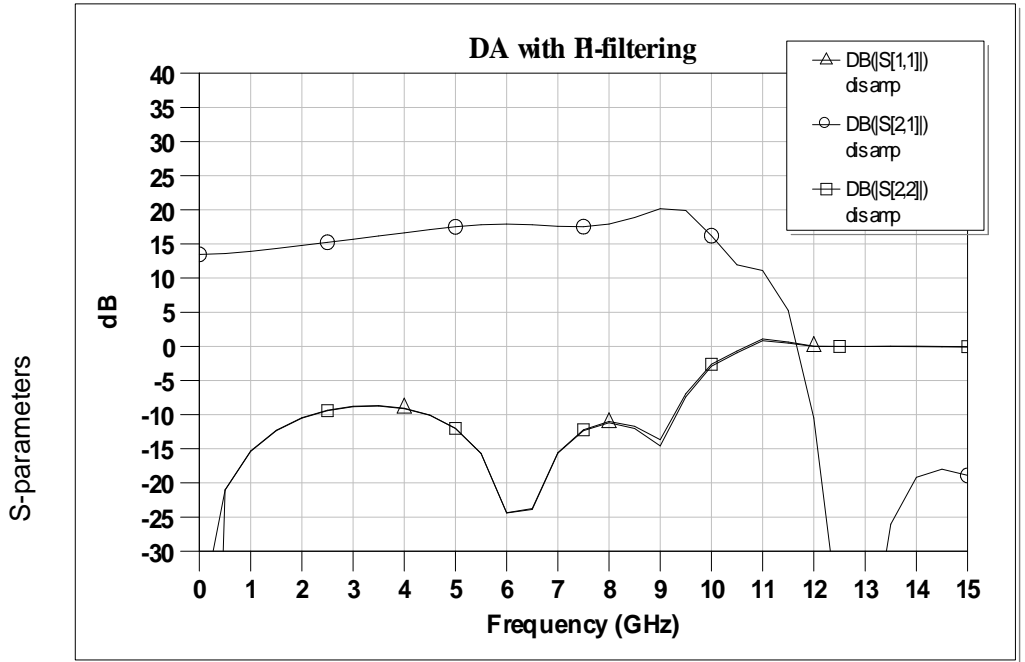

Fig.8 S-parameters of the DA based $\pi$-filtering 


\section{Conclusion}

This paper demonstrated that $\pi$-filtering structure can be used in distributed amplifiers instead of T-structure to eliminate some of their inductors and to improve the circuit performance in less layout space. Schematic amplifiers based on $\mathrm{T}$ and $\pi$-filtering sections have been designed and simulated. Simulation results verified the proposed design method.

\section{References}

[1] E. Ginzton, W. Hewlett, J. Jasberg and J. Noe. , “ Distributed Amplification”, Proc. IRE. , pp. 956-969, Aug. 1948.

[2] T. Wong, “Fundamentals of Distributed Amplification ”, Artech House, 1993.

[3] J. Aguirre and C. Plett, “ 50-GHz SiGe HBT Distributed Amplifiers Employing Constantk and m-Derived Filter Sections", IEEE Trans. Microwave Theo. Tech., , pp. 15731579, May 2004.

[4] E. Strid and K. Gleason , “A DC-12 GHz Monolithic GaAs FET Distributed Amplifier” IEEE Tran. Microwave Theo. Tech., pp. 969-975, July 1982.

[5] R. Majdi-Ahy, C.Nishimoto, M. Riaziat, M. Glenn, S. Silverman, S. Weng, Y. Pao, G. Zdasiuk, S. Bandy, and Z. Tan, "5-100 GHz InP Coplanar Waveguide MMIC Distributed Amplifier ", IEEE Tran. Microwave Theo. Tech., pp.1986-1993, Sept. 1990.

[6] B. Green, S Lee, K. Chu, K. Webb, and L. Eastman, "High Efficiency Monolithic Gallium Nitride Distributed Amplifier ", IEEE Microwave and Guided Wave Letters, pp. 270-272, July 2000 .

[7] R. Liu, C. Lin, K. Deng, and H. Wang, " Design and Analysis of DC-to-14-GHz and 22-GHz CMOS Cascode Distributed Amplifiers " , IEEE Journal of Solid-State Circuits, pp. 1370-1374, Aug. 2004.

[8] S. Mohan, M. Hershenson, S. Boyd, and T. Lee, "Simple Accurate Expressions for Planar Spiral Inductances " , IEEE Journal of Solid-State Circuits, pp. 1419-1424, Oct. 1999.

[9] J. Park, “ Design of an RF CMOS ultra-wideband Amplifier Using Parasitic - A ware Synthesis and Optimization”, Ph.D. Dissertation, University of Washington, 2003.

[10] D. Pozar, “Microwave Engineering ” $3{ }^{\text {rd }}$ Edition John Wiley and Sons, 2005. 